\title{
Die offene Lizenz: Was darf ich mit dem Lehrbuch machen?
}

Dieses Lehrbuch ist unter der Creative-Commons-Lizenz BY-SA 4.0 offen lizenziert worden. Die offene Lizenz ermöglicht es, dass die Ausführungen im Lehrbuch als Grundlage für viele weitere Open-Educational-Resources-Projekte dienen können. Professor`innen, Anwendungskursleiter ${ }^{\star}$ innen, Studierende, aber auch alle darüber hinaus Interessierten sind herzlich dazu eingeladen, das Lehrbuch zu ihren Zwecken zu nutzen, zu bearbeiten und weiterzuentwickeln.

Was bedeutet die Lizenz im Einzelnen?

\section{Das Lehrbuch darf in jedwedem Format oder Medium vervielfältigt und weiterverbreitet werden!}

Wenn ein Professor, ein*e Anwendungskursleiter*in oder ein privater Repetitor also findet, unser Lehrbuch sei so gut, dass er es allen Studierenden zur Verfügung stellen möchte: Nur zu! Jeder kann das Lehrbuch und die Abschnitte darin frei verbreiten und allen Interessierten zur Verfügung stellen.

Das Lehrbuch und Ausschnitte daraus dürfen geremixt und verändert werden und es darf auf dem Lehrbuch aufgebaut werden, und zwar für beliebige Zwecke, sogar kommerziell!

Wenn ein Professor, ein*e Anwendungskursleiter*in oder ein privater Repetitor also findet, die Ausführungen in einem Abschnitt des Lehrbuchs eigneten sich perfekt für eine eigene Falllösung, ein Skript oder eigene wissenschaftliche Ausführungen, kann er Auszüge daraus einfach verarbeiten und sie auch um eigene Ausführungen ergänzen.

Diese Rechte stehen jedoch nur unter zwei Einschränkungen offen:

Es müssen angemessene Urheber- und Rechteangaben gemacht, einen Link zur Lizenz beigefügt und angeben werden, ob Änderungen vorgenommen wurden.

Professoren, Anwendungskursleiter*innen oder Repetitoren müssen also darauf hinweisen, dass ihre Falllösung auf den Ausführungen in unserem Lehrbuch beruht und dass sie das Material verwenden, weil es unter einer offenen Lizenz veröffentlicht wurde. Außerdem müssen sie darauf hinweisen, wenn das Material verändert wurde; sie dürfen also nicht so tun, als ob ihre Ergänzungen von den Autor*innen zu verantworten wären. 
Wenn das Material geremixt, verändert oder anderweitig direkt darauf aufbaut wird, dürfen die neuen Beiträge nur unter derselben Lizenz wie das Original verbreitet werden, also unter der Creative-Commons-Lizenz BY-SA 4.0.

Wer Passagen des Lehrbuchs als Grundlage für eigene Arbeiten verwendet, muss auch seine darauf beruhende Arbeit unter der Lizenz CC BY-SA 4.0 zur Verfügung stellen. Repetitoren, Anwendungskursleiter*innen oder Professoren, die auf Grundlage unseres Lehrbuchs Fälle, Skripte oder einen Aufsatz erstellen, sind dazu verpflichtet, diesen sodann unter offener Lizenz zu veröffentlichen. Auch diese Werke stehen dann also frei für alle zur Verfügung.

Formulierungsvorschlag bei Nutzung von Textpassagen aus dem Lehrbuch:

„Dieser Text steht unter der Lizenz CC BY-SA 4.0 (https://creativecommons.org/licenses/by-sa/ 4.0/deed.de). Er beruht auf dem Werk von Autor*in, in: Eisentraut, Verwaltungsrecht in der Klausur, § \#\#, Rn. \#\# (Link zur Originalquelle), veröffentlicht unter der Lizenz CC BY-SA 4.O. Für Änderungen ist allein der/die Urheber*in dieser Überarbeitung verantwortlich.“ 